\title{
Evidence for Dominant Role of Tunneling in Condensed Phases and at High Temperatures: Double Hydrogen Transfer in Porphycenes.
}

\author{
Supporting Information \\ Piotr Ciąćka ${ }^{\mathrm{a}}$, Piotr Fita ${ }^{\mathrm{a}}$, Arkadiusz Listkowski ${ }^{\mathrm{b}, \mathrm{c}}$, Czesław Radzewicz $^{\mathrm{a}}$, Jacek Waluk ${ }^{\mathrm{b}, \mathrm{c}^{*}}$ \\ ${ }^{\text {a }}$ Institute of Experimental Physics, Faculty of Physics, University of Warsaw, Pasteura 5, 02-093 Warsaw, Poland \\ ${ }^{\mathrm{b}}$ Institute of Physical Chemistry, Polish Academy of Sciences, Kasprzaka 44/52, 01-224 Warsaw, Poland \\ ${ }^{c}$ Faculty of Mathematics and Natural Sciences, College of Science, Cardinal Stefan Wyszyński University, Dewajtis 5, \\ 01-815 Warsaw, Poland
}

\section{Experimental procedures}

Syntheses and purification procedures of both bare porphycene and 2,7,12,17-tetra-tert butylporphycene have been described earlier. ${ }^{1,2}$ The solubility of porphycenes and the availability of deuterated varieties for investigating isotope effects dictated the choice of solvents, which have been used as provided. For temperatures below $294 \mathrm{~K}$, a mixture of ethanol (POCH, sp. pure) and methanol (POCH, p.a.) or ethanol- $d_{1}$ (Sigma Aldrich) and methanol- $d_{1}$ (Armar) at 4:1 volume ratio were employed because of good glassforming capabilities down to at least $77 \mathrm{~K}^{3}$ No phase transitions occurred in the sample when cooled down, therefore the sample environment could be considered liquid at all temperatures. Its viscosity was, however, strongly temperature-dependent and rotational diffusion slowed down with lowering temperature. As a result, the rotational diffusion times were in the whole temperature range significantly longer than tautomerization times.

For temperatures above $294 \mathrm{~K}$, a mixture of toluene $(\mathrm{POCH}$, p.a) and paraffin oil (Fluka, for IR spectroscopy), was chosen because a relatively high viscosity of the solution allowed for easier separation of hydrogen transfer- and rotation-related timescales in this temperature range.

For isotope effect investigations at elevated temperatures, a mixture of methanol- $d_{1}$ and ethylene glycol- $d_{2}$ (both from Armar) was used. As confirmed by comparison of rates measured for different solvents at room temperature, the spread of which was less than three standard deviations, the choice of solvent had no influence on the tautomerization kinetics.

All the deuterated samples were stored sealed for a few days before measurements and checked periodically until no further changes in ultrafast anisotropy kinetics at room temperature were observed, a sign of a full hydrogen-deuterium exchange having taken place. Thus, it can be safely assumed that contributions of non-deuterated (HH) and singly deuterated (HD) forms in solutions are negligible.

Ground- and excited-state hydrogen transfer rates were obtained by monitoring time-dependent polarization anisotropy of transient absorption. We used our ultrafast pump-probe setup and retrieval procedures of the reaction rates from polarization anisotropy decays as described elsewhere. ${ }^{4}$ For the purpose of the present work, the setup was rebuilt to enable measurements in a wide temperature range by virtue of heated and cryogenically cooled sample compartments.

For sample heating, a custom-made PID temperature-controlled cuvette housing was built, whereas cryogenic cooling employed a helium cryostat from Advanced Research Systems model 204 A (head), ARS-4HW (compressor) and an SRS 331 S temperature controller. Those enabled us to reliably retrieve tautomerization rates in the range from $393 \mathrm{~K}$ down to around $80 \mathrm{~K}$. Prolonged exposures of bare porphycene solution to temperatures above the upper limit caused changes in stationary absorption spectra. The lower temperature limit resulted from probability of cuvette damage. Intending to keep it as low as possible, we capped most of our experiments at $80 \mathrm{~K}$, the exception being measurements of undeuterated TTPC, performed down to ca. $30 \mathrm{~K}$.

Off-the-shelf quartz cuvettes (Hellma) were sealed with silicone rubber used for mould-making, which proved a reliable seal for vacuum down to $10^{-6} \mathrm{mbar}$ at temperatures lower than those corresponding to the damage threshold of cuvettes, as well as for preventing solution evaporation at higher temperatures.

A $2 \mathrm{~mm}$ light path cuvette was used with a heater. For measurements in a cryostat, it was observed that at lower temperatures bare porphycene precipitated from a solution and either directly scattered pump light or formed nucleation centers, leading to low optical quality glass. To lower the probability of the aforementioned phenomena, a five times less concentrated porphycene solution was prepared and put in a $10 \mathrm{~mm}$ light path cuvette. Yielding the same absorbance, it brought the solution much farther away from saturation condition at lower temperatures.

Due to the higher dispersion of a long sample, time resolution was affected. Our typical pulses of $38 \mathrm{fs}$ (full width at half maximum) only broadened to $53 \mathrm{fs}$ after traversing a $10 \mathrm{~mm}$ cuvette (ethanol group velocity dispersion of $51 \mathrm{fs}^{2} / \mathrm{mm}$ was used in the calculations). Potentially more significant was the effect of temporal walk-off of pulses in two color experiments, their central wavelengths separated by approx. $70 \mathrm{~nm}$. Different group velocities led to degradation of the temporal resolution to approx. $200 \mathrm{fs}$. Expecting anisotropy dynamics to slow down at lower temperatures anyway, we deemed time resolution decrease acceptable and something that would not affect the retrieved tautomerization rates. A comparison of anisotropy decay times recorded at room temperature for both cuvettes, values of which differed by less than two standard deviations, confirmed this conclusion.

(1) N. Urbańska, M. Pietraszkiewicz, J. Waluk, J. Porphyrins Phthalocyanines 2007, 11, 596-600.

(2) I. Czerski, A. Listkowski, J. Nawrocki, N. Urbańska, H. Piwoński, A. Sokołowski, O. Pietraszkiewicz, M. Pietraszkiewicz, J. Waluk, J. Porphyrins Phthalocyanines 2012, 16, 589-602.

(3) J. Smith, J. K. Smith, S. P. McGlynn, Rev. Sci. Instr. 1962, 33, 13671371.

(4) P. Ciąćka, P. Fita, A. Listkowski, M. Kijak, S. Nonell, D. Kuzuhara, H. Yamada, C. Radzewicz, J. Waluk, J. Phys. Chem. B 2015, 119, 2292-2301. 


\section{Additional figures}

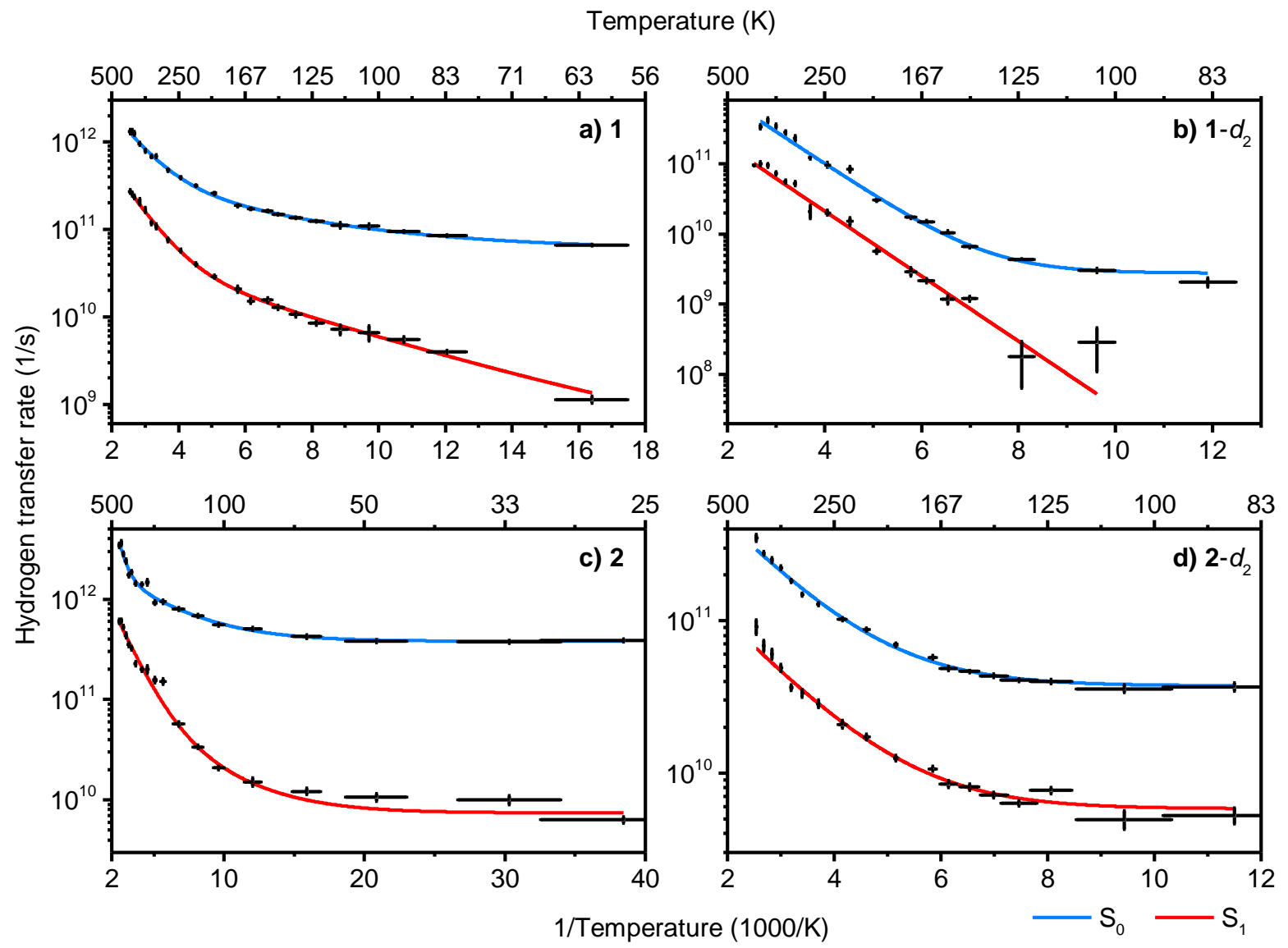

Figure 1S. Comparison of ground $\left(S_{0}\right)$ and excited state $\left(S_{1}\right)$ Arrhenius plots for tautomerization of porphycene $(\mathbf{1}$, a), 2,7,12,17-tetra-tertbutylporphycene $(\mathbf{2}, \mathrm{c})$ and their $\mathrm{N}$-deuterated isotopologues $(\mathrm{b}, \mathrm{d})$.
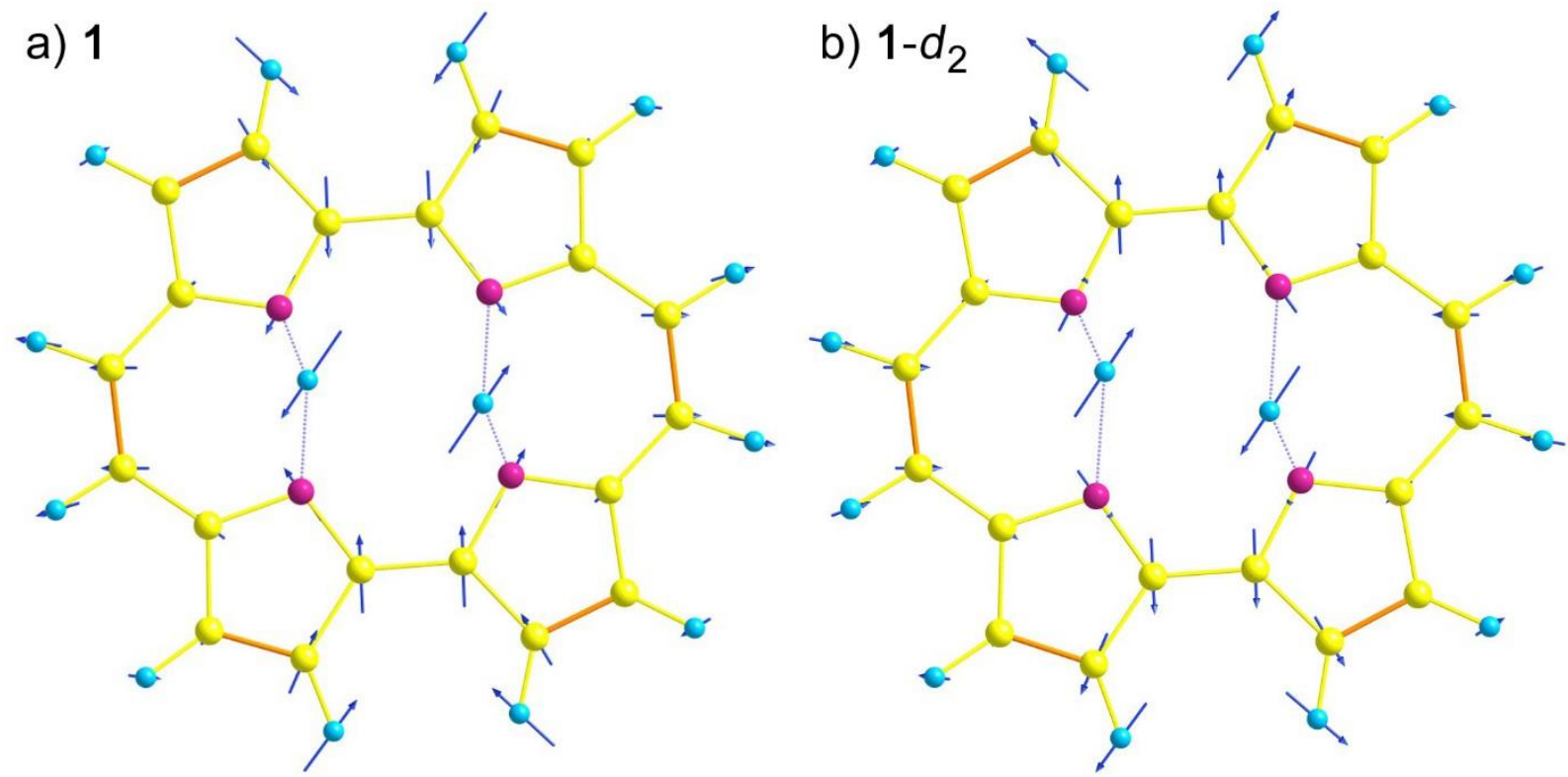

Figure 2S. Comparison of the $180 \mathrm{~cm}^{-1}$ normal modes of porphycene $(\mathbf{1}$, a) and its $\mathrm{N}$-deuterated isotopologue (b) 

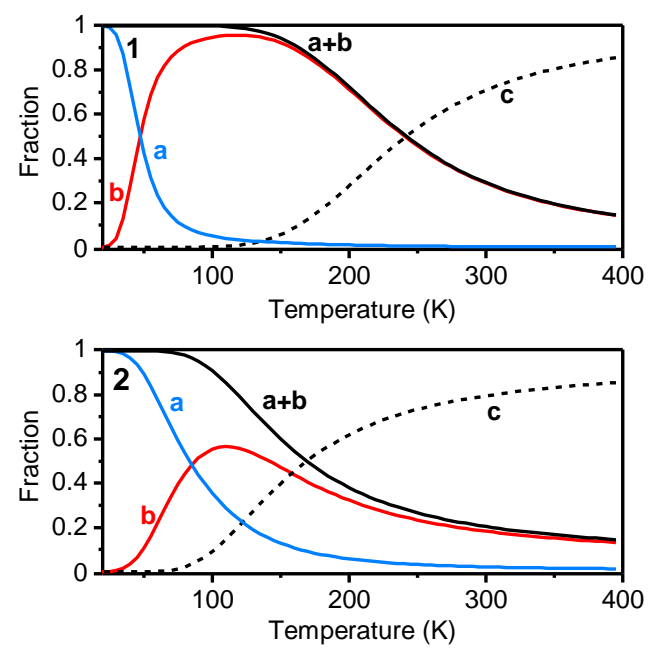

Figure 3S. Contributions of different reaction channels to the electronic excited state $\left(\mathrm{S}_{1}\right)$ tautomerization rates in $\mathbf{1}$ (top) and $\mathbf{2}$ (bottom) at various temperatures: (a) vibrational ground state tunneling; (b) vibrationally activated $\left(E_{1}=0.5 \mathrm{kcal} / \mathrm{mol}\right)$ tunneling; (c) other channels.
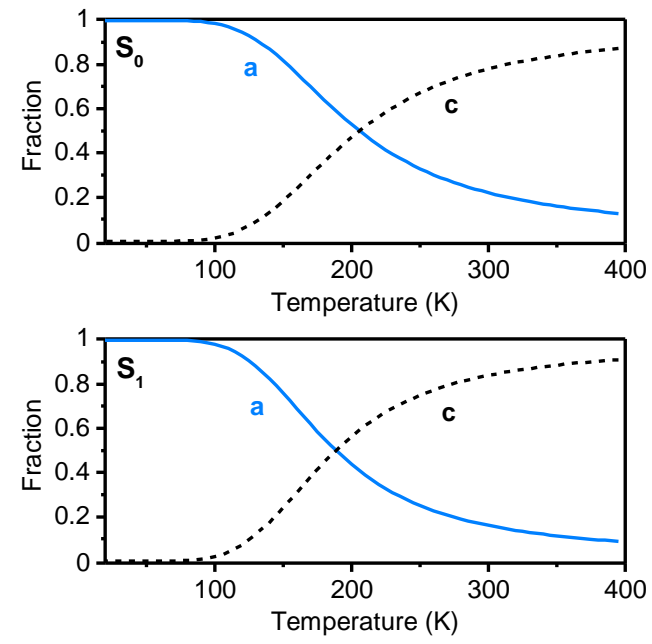

Figure 4S. Contributions of different reaction channels to the electronic ground ( $\mathrm{S}_{0}$, top) and excited $\left(\mathrm{S}_{1}\right.$, bottom) states tautomerization rates in 2- $d_{2}$ at various temperatures: (a) vibrational ground state tunneling; (c) other channels.

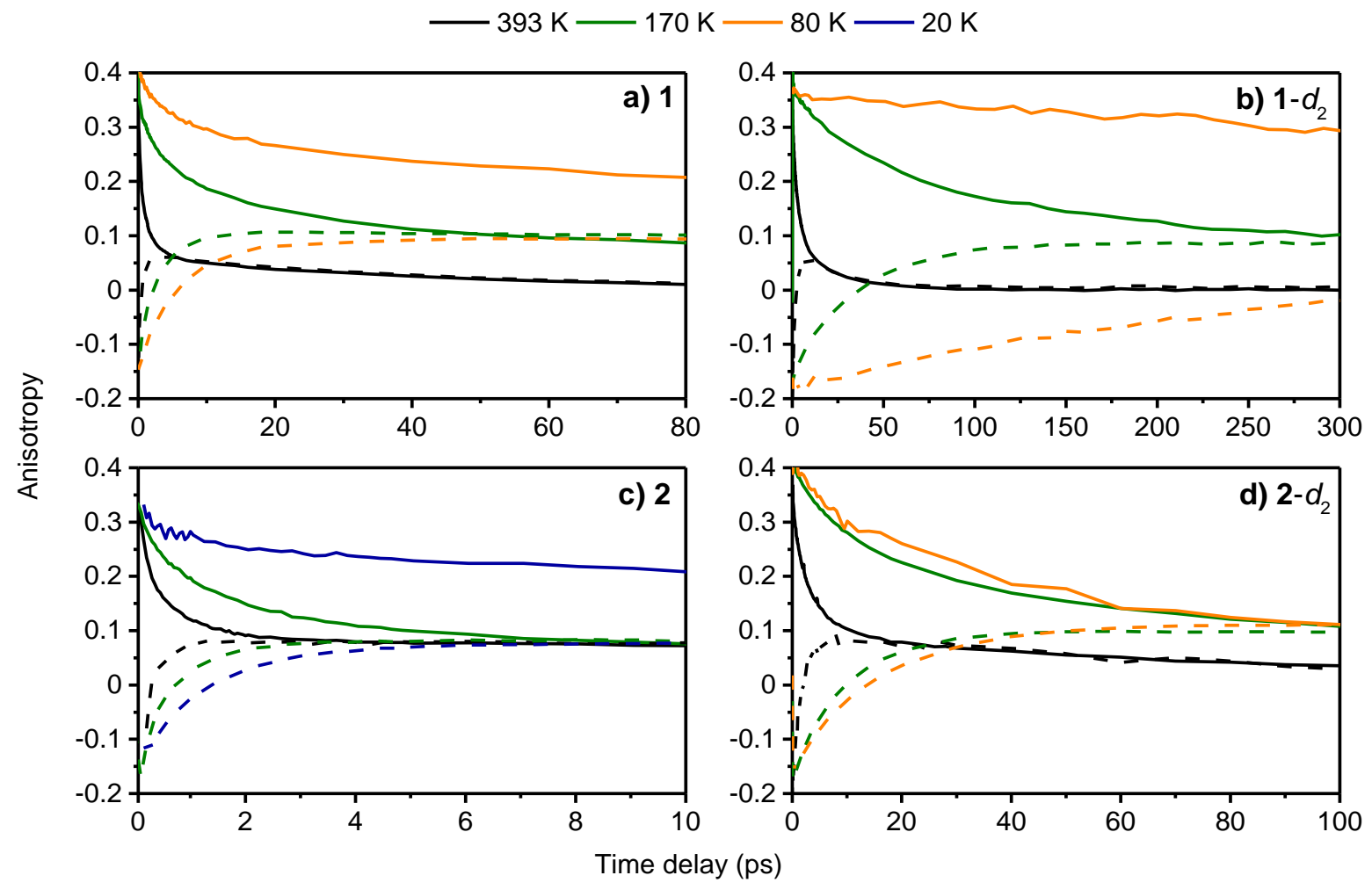

Figure 5S. Sample anisotropy kinetics recorded for 1 (a), 2 (c) and their N-deuterated isotopologues 1- $d_{2}, \mathbf{2}-d_{2}$ (b, d) with excitation to the $\mathrm{S}_{1}$ state and probing the $\mathrm{S}_{1} \leftarrow \mathrm{S}_{0}$ (solid lines) or $\mathrm{S}_{2} \leftarrow \mathrm{S}_{0}$ (dashed lines) transitions. 


\section{Tautomerization rates}

Tables $1 \mathrm{~S}$ and $2 \mathrm{~S}$ contain tautomerization rates for the ground $\left(\mathrm{S}_{0}\right)$ and the first excited singlet states $\left(\mathrm{S}_{1}\right)$ for porphycene $(\mathbf{1}), 2,7,12,17$ tetra-tert-butyl porphycene (2) and their doubly deuterated isotopologues (1- $\left.d_{2}, \mathbf{2}-d_{2}\right)$ extracted from time-resolved measurements performed at different temperatures. These data are also plotted in Figures 1 and 1S. Temperature uncertainties should be understood as maximum errors whereas rates' errors have a meaning of a standard deviation. Details of the sample environment are given above, in the section Experimental procedures.

Table 1S. Tautomerization rates of porphycene (1) and doubly N-deuterated porphycene (1- $\left.d_{2}\right)$ at different temperatures.

\begin{tabular}{|c|c|c|c|c|c|}
\hline \multicolumn{3}{|c|}{1} & \multicolumn{3}{|c|}{$1-d_{2}$} \\
\hline $\begin{array}{c}\mathbf{T} \\
{[\mathbf{K}]}\end{array}$ & $\begin{array}{c}\mathbf{S}_{\mathbf{0}} \\
{\left[\mathbf{s}^{-1}\right]}\end{array}$ & $\begin{array}{c}\mathbf{S}_{\mathbf{1}} \\
{\left[\mathbf{s}^{-1}\right]}\end{array}$ & $\begin{array}{c}\mathbf{T} \\
{[\mathbf{K}]}\end{array}$ & $\begin{array}{c}\mathbf{S}_{\mathbf{0}} \\
{\left[\mathbf{s}^{-1}\right]}\end{array}$ & $\begin{array}{c}\mathbf{S}_{1} \\
{\left[\mathbf{s}^{-1}\right]}\end{array}$ \\
\hline $393 \pm 1$ & $(13.2 \pm 0.7) \cdot 10^{11}$ & $(2.70 \pm 0.14) \cdot 10^{11}$ & $373 \pm 1$ & $(3.3 \pm 0.3) \cdot 10^{11}$ & $(10.0 \pm 0.6) \cdot 10^{10}$ \\
\hline $383 \pm 1$ & $(13.2 \pm 0.7) \cdot 10^{11}$ & $(2.53 \pm 0.12) \cdot 10^{11}$ & $353 \pm 1$ & $(4.2 \pm 0.4) \cdot 10^{11}$ & $(9.4 \pm 0.6) \cdot 10^{10}$ \\
\hline $373 \pm 1$ & $(12.5 \pm 0.7) \cdot 10^{11}$ & $(2.34 \pm 0.10) \cdot 10^{11}$ & $333 \pm 1$ & $(3.4 \pm 0.3) \cdot 10^{11}$ & $(7.2 \pm 0.4) \cdot 10^{10}$ \\
\hline $353 \pm 1$ & $(9.4 \pm 0.4) \cdot 10^{11}$ & $(2.1 \pm 0.2) \cdot 10^{11}$ & $313 \pm 1$ & $(2.8 \pm 0.2) \cdot 10^{11}$ & $(5.6 \pm 0.3) \cdot 10^{10}$ \\
\hline $333 \pm 1$ & $(7.9 \pm 0.3) \cdot 10^{11}$ & $(1.67 \pm 0.12) \cdot 10^{11}$ & $295 \pm 1$ & $(2.3 \pm 0.3) \cdot 10^{11}$ & $(5.2 \pm 0.4) \cdot 10^{10}$ \\
\hline $313 \pm 1$ & $(6.8 \pm 0.2) \cdot 10^{11}$ & $(1.19 \pm 0.06) \cdot 10^{11}$ & $270 \pm 1$ & $(1.22 \pm 0.06) \cdot 10^{11}$ & $(2.1 \pm 0.5) \cdot 10^{10}$ \\
\hline $300 \pm 1$ & $(6.8 \pm 0.3) \cdot 10^{11}$ & $(1.09 \pm 0.08) \cdot 10^{11}$ & $246 \pm 2$ & $(9.6 \pm 0.8) \cdot 10^{10}$ & $(2.0 \pm 0.2) \cdot 10^{10}$ \\
\hline $271 \pm 1$ & $(4.76 \pm 0.14) \cdot 10^{11}$ & $(7.6 \pm 0.4) \cdot 10^{10}$ & $221 \pm 2$ & $(8.3 \pm 0.7) \cdot 10^{10}$ & $(1.5 \pm 0.2) \cdot 10^{10}$ \\
\hline $246 \pm 1$ & $(3.91 \pm 0.10) \cdot 10^{11}$ & $(5.7 \pm 0.2) \cdot 10^{10}$ & $197 \pm 2$ & $(3.03 \pm 0.15) \cdot 10^{10}$ & $(5.6 \pm 0.4) \cdot 10^{9}$ \\
\hline $221 \pm 1$ & $(3.16 \pm 0.09) \cdot 10^{11}$ & $(4.0 \pm 0.2) \cdot 10^{10}$ & $173 \pm 3$ & $(1.72 \pm 0.06) \cdot 10^{10}$ & $(2.9 \pm 0.4) \cdot 10^{9}$ \\
\hline $197 \pm 2$ & $(2.58 \pm 0.07) \cdot 10^{11}$ & $(2.89 \pm 0.12) \cdot 10^{10}$ & $164 \pm 4$ & $(1.47 \pm 0.09) \cdot 10^{10}$ & $(2.16 \pm 0.14) \cdot 10^{9}$ \\
\hline $173 \pm 2$ & $(1.87 \pm 0.05) \cdot 10^{11}$ & $(2.1 \pm 0.2) \cdot 10^{10}$ & $153 \pm 3$ & $(1.04 \pm 0.07) \cdot 10^{10}$ & $(1.2 \pm 0.2) \cdot 10^{9}$ \\
\hline $162 \pm 2$ & $(1.72 \pm 0.03) \cdot 10^{11}$ & $(1.52 \pm 0.10) \cdot 10^{10}$ & $143 \pm 3$ & $(6.7 \pm 0.3) \cdot 10^{9}$ & $(1.20 \pm 0.10) \cdot 10^{9}$ \\
\hline $150 \pm 3$ & $(1.62 \pm 0.04) \cdot 10^{11}$ & $(1.56 \pm 0.10) \cdot 10^{10}$ & $124 \pm 4$ & $(4.31 \pm 0.15) \cdot 10^{9}$ & $(1.8 \pm 1.2) \cdot 10^{8}$ \\
\hline $143 \pm 3$ & $(1.47 \pm 0.03) \cdot 10^{11}$ & $(1.28 \pm 0.07) \cdot 10^{10}$ & $104 \pm 4$ & $(3.0 \pm 0.2) \cdot 10^{9}$ & $(2.9 \pm 1.8) \cdot 10^{8}$ \\
\hline $133 \pm 3$ & $(1.36 \pm 0.03) \cdot 10^{11}$ & $(1.06 \pm 0.07) \cdot 10^{10}$ & $84 \pm 4$ & $(2.1 \pm 0.3) \cdot 10^{9}$ & - \\
\hline $123 \pm 3$ & $(1.23 \pm 0.03) \cdot 10^{11}$ & $(8.5 \pm 0.5) \cdot 10^{9}$ & & & \\
\hline $113 \pm 3$ & $(1.11 \pm 0.08) \cdot 10^{11}$ & $(7.1 \pm 1.0) \cdot 10^{9}$ & & & \\
\hline $103 \pm 3$ & $(1.09 \pm 0.08) \cdot 10^{11}$ & $(6.6 \pm 1.3) \cdot 10^{9}$ & & & \\
\hline $93 \pm 4$ & $(9.5 \pm 0.2) \cdot 10^{10}$ & $(5.5 \pm 0.4) \cdot 10^{9}$ & & & \\
\hline $83 \pm 4$ & $(8.47 \pm 0.15) \cdot 10^{10}$ & $(3.97 \pm 0.13) \cdot 10^{9}$ & & & \\
\hline $61 \pm 4$ & $(6.58 \pm 0.09) \cdot 10^{10}$ & $(1.14 \pm 0.11) \cdot 10^{9}$ & & & \\
\hline
\end{tabular}


Table 2S. Tautomerization rates of 2,7,12,17-tetra-tert-butyl porphycene (2) and its doubly N-deuterated isotopologue (2- $\left.d_{2}\right)$ at different temperatures.

\begin{tabular}{|c|c|c|c|c|c|}
\hline \multicolumn{3}{|c|}{2} & \multicolumn{3}{|c|}{ 2- $d_{2}$} \\
\hline $\begin{array}{c}\mathbf{T} \\
{[\mathbf{K}]}\end{array}$ & $\begin{array}{c}\mathbf{S}_{\mathbf{0}} \\
{\left[\mathbf{s}^{-1}\right]}\end{array}$ & $\begin{array}{c}\mathbf{S}_{1} \\
{\left[\mathbf{s}^{-1}\right]}\end{array}$ & $\begin{array}{c}\mathbf{T} \\
{[\mathbf{K}]}\end{array}$ & $\begin{array}{c}\mathbf{S}_{\mathbf{0}} \\
{\left[\mathbf{s}^{-1}\right]}\end{array}$ & $\begin{array}{c}\mathbf{S}_{1} \\
{\left[\mathbf{s}^{-1}\right]}\end{array}$ \\
\hline $393 \pm 1$ & $(3.5 \pm 0.2) \cdot 10^{12}$ & $(6.0 \pm 0.3) \cdot 10^{11}$ & $393 \pm 1$ & $(3.5 \pm 0.2) \cdot 10^{11}$ & $(9.1 \pm 1.0) \cdot 10^{10}$ \\
\hline $373 \pm 1$ & $(3.6 \pm 0.2) \cdot 10^{12}$ & $(6.1 \pm 0.3) \cdot 10^{11}$ & $373 \pm 1$ & $(2.76 \pm 0.08) \cdot 10^{11}$ & $(6.8 \pm 0.6) \cdot 10^{10}$ \\
\hline $353 \pm 1$ & $(2.82 \pm 0.13) \cdot 10^{12}$ & $(5.3 \pm 0.3) \cdot 10^{11}$ & $353 \pm 1$ & $(2.50 \pm 0.09) \cdot 10^{11}$ & $(6.0 \pm 0.5) \cdot 10^{10}$ \\
\hline $333 \pm 1$ & $(2.38 \pm 0.12) \cdot 10^{12}$ & $(4.3 \pm 0.3) \cdot 10^{11}$ & $333 \pm 1$ & $(2.22 \pm 0.06) \cdot 10^{11}$ & $(4.9 \pm 0.3) \cdot 10^{10}$ \\
\hline $313 \pm 1$ & $(1.74 \pm 0.08) \cdot 10^{12}$ & $(3.5 \pm 0.2) \cdot 10^{11}$ & $313 \pm 1$ & $(1.82 \pm 0.04) \cdot 10^{11}$ & $(3.6 \pm 0.2) \cdot 10^{10}$ \\
\hline $294 \pm 1$ & $(1.85 \pm 0.07) \cdot 10^{12}$ & $(3.25 \pm 0.15) \cdot 10^{11}$ & $294 \pm 1$ & $(1.48 \pm 0.04) \cdot 10^{11}$ & $(3.3 \pm 0.2) \cdot 10^{10}$ \\
\hline $271 \pm 2$ & $(1.43 \pm 0.06) \cdot 10^{12}$ & $(2.27 \pm 0.11) \cdot 10^{11}$ & $270 \pm 1$ & $(1.28 \pm 0.03) \cdot 10^{11}$ & $(2.8 \pm 0.2) \cdot 10^{10}$ \\
\hline $242 \pm 3$ & $(1.40 \pm 0.06) \cdot 10^{12}$ & $(2.00 \pm 0.08) \cdot 10^{11}$ & $241 \pm 4$ & $(1.02 \pm 0.03) \cdot 10^{11}$ & $(2.08 \pm 0.13) \cdot 10^{10}$ \\
\hline $221 \pm 3$ & $(1.47 \pm 0.09) \cdot 10^{12}$ & $(2.0 \pm 0.2) \cdot 10^{11}$ & $217 \pm 2$ & $(8.8 \pm 0.3) \cdot 10^{10}$ & $(1.72 \pm 0.08) \cdot 10^{10}$ \\
\hline $197 \pm 4$ & $(9.3 \pm 0.4) \cdot 10^{11}$ & $(1.56 \pm 0.10) \cdot 10^{11}$ & $194 \pm 1$ & $(6.9 \pm 0.2) \cdot 10^{10}$ & $(1.25 \pm 0.06) \cdot 10^{10}$ \\
\hline $177 \pm 5$ & $(9.4 \pm 0.4) \cdot 10^{11}$ & $(1.52 \pm 0.10) \cdot 10^{11}$ & $171 \pm 2$ & $(5.75 \pm 0.13) \cdot 10^{10}$ & $(1.06 \pm 0.05) \cdot 10^{10}$ \\
\hline $148 \pm 8$ & $(7.9 \pm 0.3) \cdot 10^{11}$ & $(5.7 \pm 0.3) \cdot 10^{10}$ & $163 \pm 4$ & $(4.86 \pm 0.15) \cdot 10^{10}$ & $(8.5 \pm 0.5) \cdot 10^{9}$ \\
\hline $123 \pm 5$ & $(6.8 \pm 0.2) \cdot 10^{11}$ & $(3.38 \pm 0.14) \cdot 10^{10}$ & $153 \pm 4$ & $(4.63 \pm 0.09) \cdot 10^{10}$ & $(8.1 \pm 0.4) \cdot 10^{9}$ \\
\hline $104 \pm 4$ & $(5.6 \pm 0.2) \cdot 10^{11}$ & $(2.08 \pm 0.09) \cdot 10^{10}$ & $143 \pm 5$ & $(4.35 \pm 0.12) \cdot 10^{10}$ & $(7.1 \pm 0.4) \cdot 10^{9}$ \\
\hline $83 \pm 4$ & $(5.1 \pm 0.2) \cdot 10^{11}$ & $(1.52 \pm 0.14) \cdot 10^{10}$ & $134 \pm 6$ & $(4.07 \pm 0.10) \cdot 10^{10}$ & $(6.3 \pm 0.3) \cdot 10^{9}$ \\
\hline $63 \pm 4$ & $(4.24 \pm 0.15) \cdot 10^{11}$ & $(1.22 \pm 0.09) \cdot 10^{10}$ & $124 \pm 6$ & $(4.00 \pm 0.13) \cdot 10^{10}$ & $(7.7 \pm 0.4) \cdot 10^{9}$ \\
\hline $48 \pm 5$ & $(3.82 \pm 0.12) \cdot 10^{11}$ & $(1.06 \pm 0.10) \cdot 10^{10}$ & $106 \pm 10$ & $(3.6 \pm 0.3) \cdot 10^{10}$ & $(5.0 \pm 0.7) \cdot 10^{9}$ \\
\hline $33 \pm 4$ & $(3.76 \pm 0.12) \cdot 10^{11}$ & $(1.0 \pm 0.1) \cdot 10^{10}$ & $87 \pm 10$ & $(3.7 \pm 0.3) \cdot 10^{10}$ & $(5.3 \pm 0.7) \cdot 10^{9}$ \\
\hline $26 \pm 4$ & $(3.88 \pm 0.13) \cdot 10^{11}$ & $(6.3 \pm 0.5) \cdot 10^{9}$ & & & \\
\hline
\end{tabular}

